\title{
Wozu Goethe?
}

\author{
Erhard Taverna
}

Die Vortragsbesucher auf der Terrasse im dritten Stock des Kantonsspitals geniessen den wolkenlosen Frühlingstag, den Blick auf die ersten Blüten der Grünanlagen, auf die Turmgiebel der Altstadt und auf das Panorama des in der Aprilsonne gleissenden, schneebedeckten Pilatus. Das Symposium «Literatur und Medizin» in Luzern, entstanden in Zusammenarbeit mit der GoetheGesellschaft Schweiz, der Thomas-Mann-Gesellschaft Schweiz und der Vereinigung Schweizer Schriftsteller-Ärzte, hat viele Neugierige, überwiegend ältere Semester, angelockt. Am Vorabend hatte Klara Obermüller im marianischen Saal den Vortragszyklus mit ihren Gedanken zum Verhältnis von Literatur und Medizin eröffnet. Heute gilt ein erster Schwerpunkt den erwähnten Klassikern, ein zweiter am nächsten Tag der Poesie- und Bibliotherapie, nachmittags abgerundet mit einem Exkurs über Gottfried Benn und Beiträgen der Association Suisse des Ecrivains Médecins (ASEM).

\section{Goethe \& Co.}

Eingerahmt von Tschaikowskys Kammermusik «Souvenirs de Florence», hatte sich Klara Obermüller zu den TV-Serien von Dr. Frank und Schwester Stephanie bekannt, zu ihrem Favoriten «Chicago Emergency», zu den populären Schicksalsorten mit dem stethoskopbewehrten Personal der coolen Drahtzieher. Jetzt blendet die Einführung zurück zum strengen Apoll, ausgerüstet mit Leier und Lorbeerkranz, zu den Säulenhallen des Heilschlafs in Epidauros. Die Dozentin, Professor Herwig aus Düsseldorf, erinnert an Goethes viele Patienten, an den suizidalen Werther, die melancholische Aurelie, den paranoiden Tasso, an die hysterische Lydie, an Mignon mit dem gebrochenen Herzen und an den depressiven Faust. Der wachstumsgestörte, liebeskranke Wilhelm Meister gerät ihr zum Lehrstück für Verwundung und Genesung, für eine lebenslängliche Therapie aus Ortsveränderungen, neuen Bezugspersonen, neuer Lektüre und heilsamer Wiederkehr des Verdrängten im Theaterspiel dank der finanziellen Unabhängigkeit durch väterliches Erbe. Auf der Strecke bleiben die geopferten Frauen, gesunden wird der tätige Mann. Frank Nager, Spezialist für internistische und metaphorische Herzen, schildert das koro- nare Leiden und die Infarkte des stets schwierigen Patienten aus Weimar, der seine Hausärzte mit ihrem Aderlass und Kampfer als Jesuiten beschimpfte und nach einer napoleonischen Kur verlangte. Das gleichnishafte Herz als Instanz gegen die «verwirrenden Lehren zum verwirrenden Tun» hat beim Dichter das Primat über den Verstand. Wer die Weisungen seines Herzens missachtet, wird krank, Herzensscharfsinn (Pascals «raison du cœur») und Herzenserleuchtung sind die Schlüsselbegriffe im Widerstreit mit einseitigem Bücher- und Faktenwissen. Famulus, dem trockenen Schleicher, fehlt die Liebe zur Schöpfung, die gefühlvolle Inspiration. Goethe praktiziert seine Forschung als Wissenschaft plus Kunst: «Das Pflanzenreich reisst in meinem Gemüte.» Ärzte sollen scharfsinnig denken und inbrünstig fühlen. Die Forderung nach einem Herzen als Wesenskern eines inneren Zentrums mache Goethe zum Lehrmeister aller Epochen.

Dem Anthroposophen Dr. Heusser aus Bern gerät die Farbenlehre zum Angelpunkt seiner Kritik an einer Wissenschaft, die das SeelischGeistige ausklammere. Eine ganzheitliche Medizin sei nur möglich, wenn sie sich nach Goethe neu ausrichte: «[...] dann hat er die Teile in der Hand, fehlt nur das geistige Band.» Der Forscher muss die Synthese anstreben und im äusseren Phänomen die innere Urform erblicken. Kants Kritik der reinen Vernunft mag er nicht gelten lassen, denn das Objektive in der Natur existiere unabhängig vom Menschen und warte auf seine Entdeckung.

Untermalt mit Bildern von Caspar David Friedrich, werden Krankheit und Heilung bei Thomas Mann mit Textanalysen aus unterschiedlichen Zeiten seines Schaffens untersucht. Es sind vor allem die Familiengeschichte der «Buddenbrooks» aus Lübeck, die Sanatoriumswelt in Davos des «Zauberbergs» und die späte Erzählung «Die Betrogene» (1952), in der die hormonellen Wirkungen eines unerkannten Uteruskarzinoms zu einem vermeintlichen zweiten Frühling verhelfen. Von Engelhardt, der Professor für Medizingeschichte, betont bei Mann die Krankheit als physische, psychische, soziale und geistige Realität. Die Biologie wird subjektiv erlebt, im Zauberberg, dem Panoptikum aller Gefühle, geht jeder anders mit seiner Krankheit um. Es gibt die Paradoxie der «sanitas perniziosa» und der «infirmitas salubris». Der Arzt, Geheim- 
rat Behrens: «Die Krankheit muss den Menschen klug und fein und besonders machen.» Die Literatur erweitert den Blick im Sinn des antiken Kausalitätsbegriffes. Dessen «causa» unterscheidet die Wirkungsursache (efficiens), zum Beispiel die Infektion mit Tbc, den Zweck (finalis), der darin besteht, dass der Kranke einen Sinn unterstellt, den Stoff (materialis), die persönliche Wahrnehmung und die Form (formalis), die Materie. Der Kritik von seiten der «Deutschen Medizinischen Wochenschrift» begegnete Mann 1925 mit dem Argument, dass beide, Literatur und Medizin, das gemeinsame Ziel der Wiederherstellung der menschlichen Einheit verfolgten.

\section{Warum nicht die Gegenwart?}

Haben uns die Ärzte von Goethe und Mann noch etwas zu sagen? Beim einen betreten sie immer die Lebensbühne, wenn eine entscheidende Wende eintritt, beim anderen sind sie meist überfordert, resigniert, delegierend oder distanziert-zynisch. Etwa der Kollege Behrens im Zauberberg: Bei ihm wird weggestorben, abgetanzt und abgeschnappt, «ich kenne den Tod, man überschätzt ihn», der Chirurg operiert, «ut aliquid fieri videatur», er tut als ob. «Ich bin der Meinung, dass es Krankheiten gibt, die für den Arzt zu gut sind», sagt Thomas Mann einmal. Der anthroposophische Beitrag zur Goetheschen Farbenlehre geriet zur einseitigen Wissenschaftskritik. Der Meister erkannte, dass die Wahrnehmung von Licht nicht durch ein Prismenexperiment zu erklären war, und bekannte, dass er «das ekelhafte Newtonische Weiss mit Gewalt verfolgte». Ohne die späteren Kenntnisse der Neu- rophysiologie musste er sich in eine Pseudolehre, in eine Farbtheologie verirren. Das tut seinen Talenten keinen Abbruch. Es wäre aber fruchtbarer gewesen, seine spekulative Methode mit der geduldigen und reduktionistischen Arbeit von mehreren Generationen Medizinern, Physiologen und Physikern zu vergleichen, bis hin zum genialen Erfinder der Polaroidkamera, Edwin Land, dem eine objektive Erklärung, eine Gesamtschau, für die bisher unverständlichen Phänomene des Farbsinns gelang. Wenn das Feindbild von der angeblich mechanistischen Weltsicht der Moderne 170 Jahre Wissenschaftsgeschichte ausblendet, sei die Frage erlaubt, was uns die Ärzte und die Patienten zur Zeit unserer Ururgrossväter noch zu sagen haben. Ist die Lektüre ihres Wirkens mehr als ein Denkmal, mehr als eine vergängliche Anekdote, mehr als langweiliger Pflichtstoff? Finden wir darin Antworten oder wenigstens Hilfen zur Bewältigung unserer Probleme? Das Fazit der Vorträge bleibt ambivalent: Grosse Literatur ist immer lesenswert, doch auch ihre Inhalte werden Geschichte. Was sie so überzeugend darlegte, das Soziale und Geistige, hat sich, zwei Weltkriege später, von Grund auf verändert. Mit Schnitzler, Döblin und Benn wäre eine Brücke in die Gegenwart gelungen. Eine Gegenwart, die reich ist an Autoren mit aktuellen literarischen Bezügen zur Jetztzeit. Eine Gegenwart der letzten 50 Jahre, die das von den (ungelesenen?) Bildungsikonen der Vergangenheit Gedachte aufs talentierteste, vielfältigste und farbigste für die weit komplexere Moderne fortentwickelt hat.

Vielleicht wird das Symposium $2005 \mathrm{zu}$ «Philosophie und Medizin» näher am Puls der Zeit sein. 